\title{
Diet matrix of stored proprietary feeds: Implications on growth response, health status and carcass yield of broiler chickens
}

Fafiolu, A. O. and *Alabi, J. O.

World Bank Centre of Excellence in Agricultural Development and Sustainable

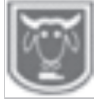

Abstract

Environment (CEADESE), Federal University of Agriculture, Abeokuta, PMB 2240, Ogun State 110001, Nigeria

*Corresponding author: joelalabi@gmail.com; +2347066486949

This study examined growth responses, health status and carcass yield of broiler chickens from 22 to 42 days of age provided with proprietary finished feeds (4 brands; $P, Q, R \& S$ ) exposed to two storage conditions (lit or dark room) for four weeks. Four hundred and eighty chicks (802.7 $\pm 14.3 \mathrm{~g})$, at 21-day-old, were randomly allocated to eight groups of 60 chicks each (6 replicates; 10 birds each). Broiler chickens were fed one of the eight resultant diets from storage exercise for $21 \mathrm{~d}$. Results revealed that performance of finishing broiler chickens were influenced by both the main and interaction effects offeed brands and storage condition. Chickens fed feeds stored in dark room gained $5.39 \%$ heavier weight with better FCR $(5.07 \%)$ and higher $(P<0.001)$ crude fat $(2.97 \%)$, protein $(2.36 \%)$, fibre $(2.43 \%)$ and ash (3.89\%) digestibilities compared to birds fed feeds stored in lit room. Chickens fed with feed $R$ had higher $(P<0.05)$ PCV, RBC, total protein, albumin, and uric acid. Duodenal and ileal villus height $(V H)$ and villus to crypt ratio (VCR) were higher in broilers fed feed $R$ stored in dark room, whereas jejunal VH and VCR were higher in birds fed feed $Q$ stored in dark room. Salmonella and lactobacillus counts were greater in birds fed feeds $R$ and $S$ stored in lit room, respectively. Higher $(P=0.008)$ dressed yield was obtained in broilers fed feed $S$ stored in lit room. The study revealed that exposure of similar or different finished feeds to varying storage conditions would impart broiler's performance differently.

Keywords: Feed technology, Diet matrix, Finished feeds; Feed storage, Performance, Chickens

\section{Introduction}

Diet matrix (otherwise refer to as Feed matrix) are complex multi-component systems which involves the nutrient and non-nutrient components of feed (water, protein, carbohydrates, lipids, fibre, minerals, vitamins, polyphenols, and a host of other compounds) and their molecular relationships to each other (Figure 1). Particle size reduction and homogenous mixing during feed production is presumed to increase the surface area of feedstuffs for enhanced enzymatic and chemical degradation in the GIT resulting to improve bioavailability (Amerah et al., 2007). However, the biochemical reactions which occur among the nutrients in the feed could alter its bioactive properties and could also elicit formation of complexes thereby having significant influence on its digestion, absorption and utilization process.

Evidently, it is a common practice for compounded feeds (either commercially prepared or on-farm formulated) to undergo storage prior to its utilization on the farm (Alabi et al., 2017). The storage conditions and duration of conservation however depends on several factors. While some farmers store their feeds in silos, others usually stacked the bagged feeds on pallets in the store house. Also, it is worthy of note that most small-scale farmers purchase finished feeds from distribution outlets, 
Diet matrix and broiler chicken performance

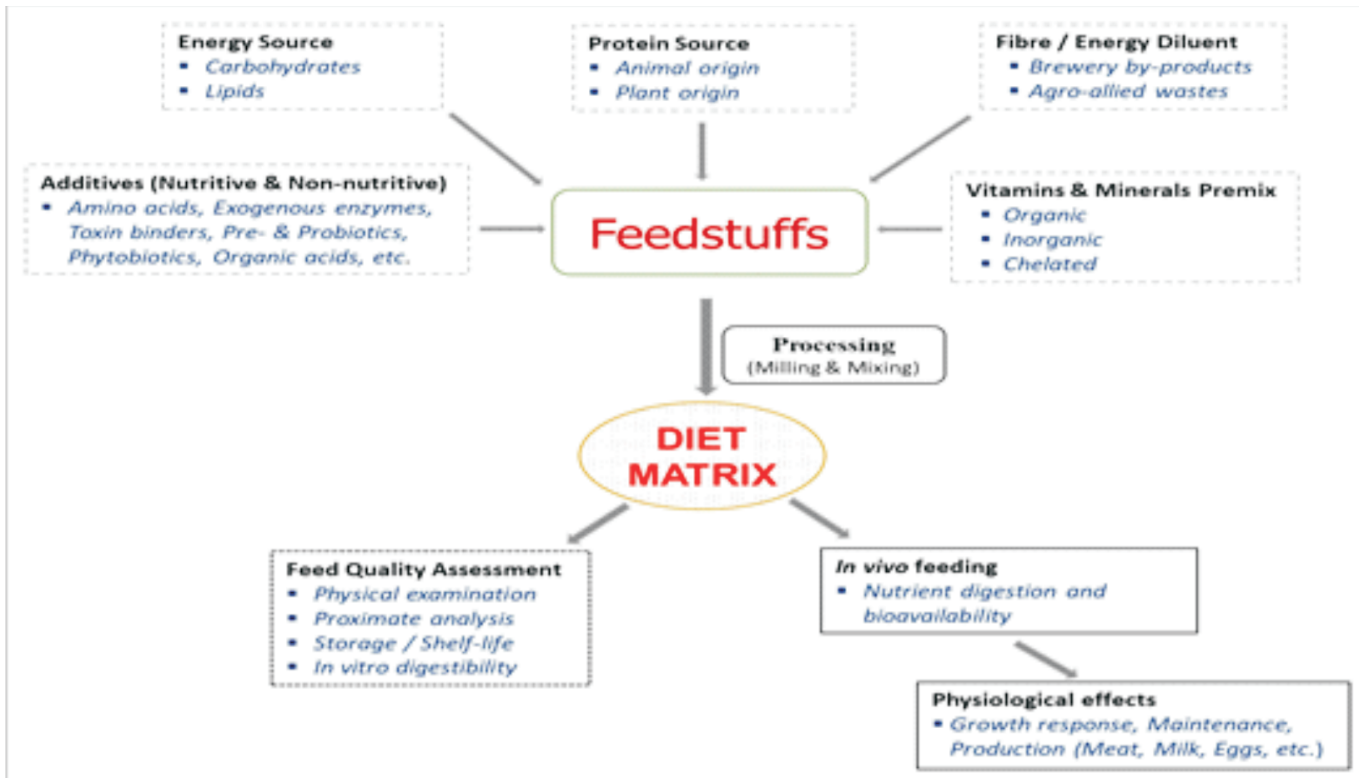

Figure 1: Feed matrix showing sources of nutrients and quality assessment

feed depots, or toll millers. The quality of which might not be ascertained owing to the storage conditions the feeds have been exposed to (Alabi et al., 2017). Improper handling of compounded feeds, by the farmers or feed distributors, before it is being fed to the poultry birds could results to feed value deterioration thereby adversely affecting the growth performance and immune status of the animals (Reddy, 2001; Yegany et al., 2002). Feed spoilage can be aggravated by a number of factors which include exposure to light, cold, heat, oxygen, moisture, enzymes, micro-organisms (bacteria, yeasts and moulds), and industrial contaminants. More so, length of storage, infestation by insects, parasites and rodents also contribute to this menace (Waheed et al. 2004; Reddy, 2001). Most stored feeds, however, undergo chemical changes as a result of increased activity of endogenous and exogenous enzymes, metabolic activity of insects and oxidation of labile ingredients such as Vitamins $\mathrm{A}, \mathrm{E}$ and unsaturated fatty acids which could alter its flavour, colour, aroma, texture, as well as the nutritive value (Uppal et al., 2004). This will consequently affect the production performance. Numerous studies have investigated the role of individual nutrients on productivity and physiological response of broiler chickens. Meanwhile, studies on the impact of diet matrix of finished feeds stored over a period of 4 weeks, either in lit or dark room, on the growth, health status and carcass yield of broiler chickens are limited. In view of this, this present study was conducted to evaluate response of finishing broiler chickens to stored finished feeds from different feed manufacturers in terms of growth performance, blood profile, intestinal morphology and carcass yield.

\section{Materials and methods Ethical approval and study location}

This study protocol and procedures received approval from the Animal Care and Use Review Committee guidelines of the College of Animal Science and Livestock Production, Federal University of Agriculture, Abeokuta, Nigeria (ACURC/2017/17/FUNAABNG). The 


\section{Fafiolu and Alabi}

feed storage and biological experiment were conducted at the Poultry Unit of the Teaching and Research Farms, Federal University of Agriculture, Abeokuta, Nigeria (Latitude $7^{\circ} 13^{\prime} 35.48^{\prime \prime} \mathrm{N}$ and Longitude $\left.3^{\circ} 25^{\prime} 39.01^{\prime \prime} \mathrm{E}\right)$ between the month of March and May, which marks the end of dry season and onset of rainy season (Google Earth Imagery, 2019). Mean ambient temperature and relative humidity during this period ranged from $31-32^{\circ} \mathrm{C}$ and $65-73 \%$, respectively. The daily chance of precipitation increased from 20 to $69 \%$ while the average rainfall recorded is between 151.50 and $173.80 \mathrm{~mm}$ (World Weather, 2019).
Storage of finished feeds

Four brands of broiler finisher diets (all in mash form) that are commonly patronized by Poultry farmers in Nigeria were studied. They were purchased from feed depots in Abeokuta, Ogun State. Efforts were made to ensure that selected feeds were obtained within 5 days of production. They were designated as $\mathrm{P}, \mathrm{Q}, \mathrm{R}$, and $\mathrm{S}$. The nutrient profile of proprietary broiler finisher diets used in this study are shown in Table 1 . The trade name of feeds investigated is not specified in this report for obvious reasons. The storage procedure used was as described by Alabi et al. (2017). $400 \mathrm{~kg}$ each of the selected feeds were purchased based on the projected feed intake.

Table 1: Nutrient profile of proprietary broiler finisher diets used (\%) ${ }^{1}$

\begin{tabular}{lcccccc}
\hline $\begin{array}{l}\text { Feed } \\
\text { brands }\end{array}$ & $\begin{array}{c}\text { Metabolizable } \\
\text { energy (Kcal/kg) }\end{array}$ & $\begin{array}{c}\text { Crude } \\
\text { protein }\end{array}$ & $\begin{array}{c}\text { Crude } \\
\text { fats }\end{array}$ & $\begin{array}{c}\text { Crude } \\
\text { fibre }\end{array}$ & Calcium & Av. Phos. \\
\hline P & 3000.00 & 20.00 & 10.00 & 10.00 & 1.00 & 0.40 \\
Q & 3000.00 & 19.00 & 6.00 & 5.00 & 1.00 & 0.40 \\
R & 2900.00 & 21.00 & 10.00 & 9.00 & 1.00 & 0.45 \\
S & 3050.00 & 19.50 & 3.00 & 5.00 & 1.00 & 0.50 \\
\hline
\end{tabular}

${ }^{1}$ Declared values by feed manufacturers; Av. Phos. $=$ Available phosphorus.

Thereafter, they were divided into two groups (200 kg each) and each group was kept in either lit room or dark room for 4 weeks before they were used as experimental diets for feeding trial. The lit room is a normal daylight room which received $12 \mathrm{hrs}$ light and $12 \mathrm{hrs}$ darkness with no artificial light while the dark room had neither natural nor artificial light. During this period, the temperature and relative humidity of the stores were monitored with digital thermometer and hygrometer (HTC 1 Brand, Yueqing Xinyang Automation Equipment Co. Ltd., China). The length of storage (4wk) adopted was based on previous findings by Alabi et al. (2017) who found that feeds retained their physical attributes with minimal weight loss up to $28^{\text {th }} \mathrm{d}$ of storage. The mean temperature and relative humidity recorded for lit room $\left(27.13^{\circ} \mathrm{C}\right.$ and $78.98 \%)$ and dark room $\left(26.84^{\circ} \mathrm{C}\right.$ and $79.75 \%)$ during storage did not differ $(P>0.05)$ significantly (Figure 2$)$.

\section{Design of the study and management of birds}

The biological experiment was undertaken in a $4 \times 2$ factorial completely randomized design (CRD) with Marshall Broiler chicks obtained from Obasanjo Farms Ltd, Ibadan, Nigeria. Prior to the feeding trial, the chicks $(n=510)$ were brooded and reared on a deep litter intensive housing unit using dried wood shavings as bedding material. Broilers were fed with basal diets formulated (ME: $3050 \mathrm{Kcal} / \mathrm{kg}$; CP: 22.50\%, EE: 3.50\%; CF: 3.75\%). All chickens were vaccinated following standard protocol while feed and clean water were supplied ad libitum. At $d 21,480$ broiler chicks of similar weights (mean weight $=802.7 \pm 14.3 \mathrm{~g}$ ) were randomly 
Diet matrix and broiler chicken performance

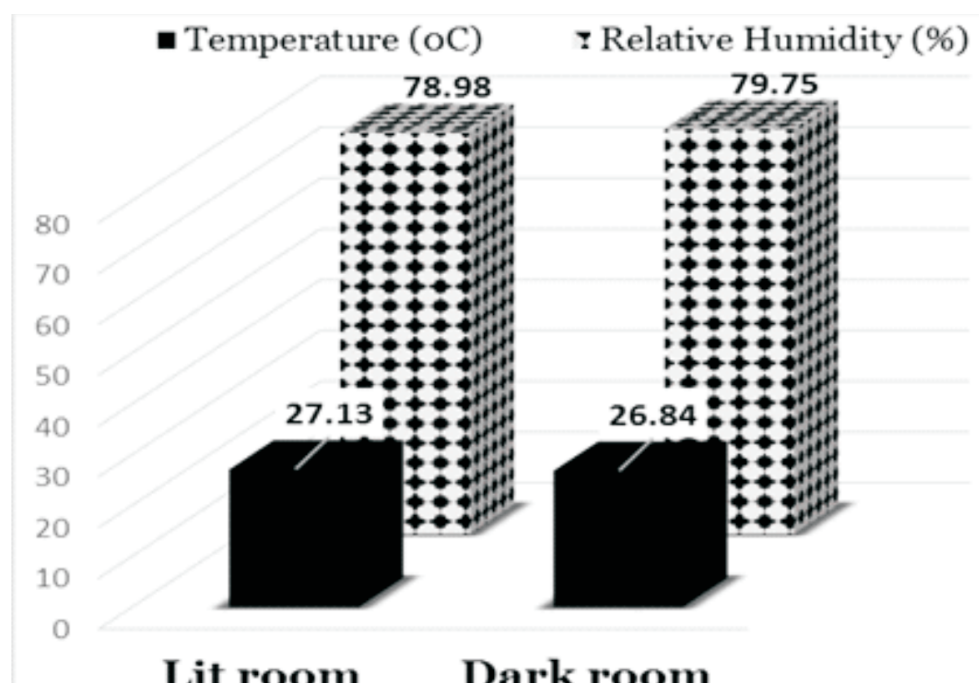

\section{Lit room Dark room}

Figure 2: Mean ambient temperature and relative humidity of storage room

assigned, on weight equalization, into 8 treatments (6 replicates of 10 birds each). Thereafter, the feeding trial commenced (on d 22) and lasted for $21 \mathrm{~d}$. Chickens were fed one of the eight resultant diets from storage exercise. Throughout the rearing period, feed and clean water were offered without restriction.

\section{Growth performance and nutrient retention}

Body weight of the chickens per replicate were recorded in the morning before feeding. Feed consumption was measured as the difference between the feed offered and leftovers on weekly basis. Mortality was noted as it occurred to determine livability. Feed conversion ratio was computed as the ratio of feed consumed to weight gain, and corrected for mortality (Muñoz et al., 2018; Craig et al., 2019). A metabolic trial was conducted for 4 days $(38-42 \mathrm{~d})$ in order to determine nutrient digestibility (Akit et al., 2019; Craig et al. 2019). Excreta collected daily were weighed and dried in vacuum oven. Feed samples and excreta collected were analysed for proximate composition using standard procedures (AOAC, 2000).

\section{Haematological and serum biochemistry indices}

At the end of the feeding trial (d 42), blood samples were collected from selected chickens (12 broilers per treatment) through the jugular vein into sampling bottles with and without anti-coagulant (EDTA) for the determination of haematological indices and serum biochemical analysis, respectively. Haematology parameters including Packed cell volume (PCV), Red blood cell (RBC), White blood cell (WBC) and differential leukocytes counts were determined according to the standard procedures for avian (Wakenell, 2010; Fafiolu et al., 2014). Serum was separated by centrifugation $(3,000 \mathrm{xg})$ for $10 \mathrm{~min}$ at $4^{\circ} \mathrm{C}$, stored at $-20^{\circ} \mathrm{C}$ before analysis. Serum levels of total protein, albumin, globulin, uric acid, creatinine and liver enzymes activities (aspartate aminotransferases, AST and alanine aminotransferases, ALT) were determined using colorimetric diagnostic kits (Labtest Diagnostica ${ }^{\circledR}$, Lagoa Santa, MG, Brazil) in a spectrophotometer (Bio-Rad Laboratories, India) with light wavelength adequate for each test. 


\section{Fafiolu and Alabi}

\section{Intestinal morphology}

At day 21, 12 broilers per treatment were selected, sacrificed by cervical dislocation, and gastrointestinal tract was carefully excised for morphological studies. Representative samples (about $3 \mathrm{~cm}$ in length) from the middle part of each of the three intestinal segments (duodenum, jejunum and ileum) were processed following the method described by Adeleye et al. (2018) and Akit et al. (2019). Intestinal villi with their crypts were individually separated under a dissecting microscope. The length and width of the villi were measured using a Nikon Phase Contrast Microscope Integrated Digital Imaging Analyses (Nikon Tec, Shinagawa, Tokyo, Japan). The villi length was measured from the tip to the villi base, and the crypt depth was measured from the base of the villi to the base of the crypt. The ratio of villus height to crypt depth (VCR) was then estimated (Fafiolu et al., 2020).

\section{Gut microbiology}

Fresh digesta from the ileum and a pair of caeca of the selected broiler chicks were collected and emptied into labelled sterile bottles for the estimation of gut microbiota (Oso et al., 2017; Akit et al., 2019). Briefly, $1 \mathrm{~g}$ of sample was dispersed in a $9 \mathrm{~mL}$ phosphate-buffered saline solution with 0.5 $\mathrm{g} / \mathrm{L}$ of $\mathrm{Cys} . \mathrm{HCl}$, and further diluted serially to a factor of $10^{-8}$. Bacteria were enumerated with $0.1 \mathrm{~mL}$ of diluted sample which was spread onto Petri dish containing selective media. Estimation of total bacteria, coliforms, salmonella, lactobacilli and clostridium counts were carried out using appropriate agar. Microbial counts were expressed as colony-forming units (CFU) of microorganism per gram of fresh sample.

\section{Carcass yield evaluation}

Twelve chickens per treatment ( 6 per sex) of nearly average weight per treatment were selected, fasted for $6 \mathrm{~h}$, and re-weighed prior to slaughter on the $42^{\text {nd }}$ day. Chickens were slaughtered, defeathered and dressed following standard commercial procedures (Oso et al., 2017). The dressed carcass and primal-cut parts including breast, drumstick, thigh, and gizzard (without content) were weighed individually using a sensitive digital electronic weighing scale (Goldline ${ }^{\circledR}$-GLF3; Goldline, Delhi, India) and expressed as percentage of respective edible carcass weight.

\section{Statistical analysis}

Data generated from this study were analysed using Analysis of Variance (ANOVA) technique in a $4 \times 2$ factorial design using GLM procedure of SAS for Windows, 9.1.3 portable version (Statistical Analysis System, 2007; Cary, NC, USA). Differences between treatment means were determined by Tukey's HSD test at $P<0.05$ level of significance. The statistical model used is as shown below:

$Y_{i j k}=\mu+F_{i}+S_{j}+\left(F^{*} S\right)_{i j}+\sum_{i j k}$

where $\mathrm{Y}_{\mathrm{ijk}}=$ Observed value / Output, $\mathrm{F}_{\mathrm{i}}=$ Effect of $i^{\text {th }}$ feed brands ( $\left.i=P, Q, R \& S\right), S_{j}=$ Effect of $j^{\text {th }}$ storage conditions $(j=$ lit room \& dark room), $\left(\mathrm{F}^{*} \mathrm{~S}\right)_{\mathrm{ij}}=$ Interaction between $\mathrm{i}^{\text {th }}$ feed brands and $\mathrm{j}^{\text {th }}$ storage conditions, and $\Sigma_{\mathrm{ijk}}=$ Random residual error.

\section{Results}

Growth performance results revealed that weight gain, feed intake and FCR differed significantly $(\mathrm{P}<0.05)$ due to interaction between feed brands and storage conditions (Table 2). Broilers fed feed S stored in dark room had $(\mathrm{P}=0.003)$ higher weight gain while those fed feed $\mathrm{S}$ stored in lit room had higher $(\mathrm{P}=0.003)$ feed intake and poorest $(\mathrm{P}=0.015)$ FCR. Main effects of feed on the 


\section{Diet matrix and broiler chicken performance}

chickens showed that broilers fed feed Q recorded higher $(\mathrm{P}=0.009)$ weight gain while those fed feed $\mathrm{R}$ had lower $(\mathrm{P}=0.004)$ feed consumption with better $(\mathrm{P}=0.020)$ feed utilization efficiency. Broilers fed feeds stored in dark room gained 5.39\% heavier ( $\mathrm{P}=0.004)$ weight, consumed less $(\mathrm{P}=0.002)$ feed, with $5.07 \%$ better $(\mathrm{P}=0.001)$ feed conversion compared to broilers fed feeds stored in lit room. Livability was not significantly $(\mathrm{P}>0.05)$ affected by the dietary factors both at main and interaction levels.

Table 2: Growth performance of broiler chickens as influenced by feeding stored finished feeds

\begin{tabular}{|c|c|c|c|c|c|}
\hline Feed brands & $\begin{array}{l}\text { Storage } \\
\text { condition }\end{array}$ & $\begin{array}{l}\text { Weight gain } \\
(\mathrm{g})\end{array}$ & $\begin{array}{c}\text { Feed intake } \\
(\mathrm{g})\end{array}$ & $\begin{array}{l}\text { FCR } \\
(\mathrm{g}: \mathrm{g})\end{array}$ & Livability (\%) \\
\hline \multirow[t]{2}{*}{$\mathrm{P}$} & Lit room & $54.77^{\mathrm{cd}}$ & $118.98^{\mathrm{abc}}$ & $2.12^{\mathrm{b}}$ & 97.50 \\
\hline & Dark room & $57.58^{\mathrm{b}}$ & $119.32^{\mathrm{abc}}$ & $2.10^{\mathrm{b}}$ & 100.00 \\
\hline \multirow[t]{2}{*}{ Q } & Lit room & $57.56^{\mathrm{b}}$ & $118.41^{\mathrm{abc}}$ & $2.09^{\mathrm{b}}$ & 97.50 \\
\hline & Dark room & $57.91^{b}$ & $120.54^{\mathrm{abc}}$ & $2.13^{\mathrm{b}}$ & 97.50 \\
\hline \multirow[t]{2}{*}{$\mathrm{R}$} & Lit room & $54.53^{\text {cd }}$ & $101.42^{\mathrm{c}}$ & $1.87^{\mathrm{d}}$ & 97.50 \\
\hline & Dark room & $55.30^{c}$ & $109.30^{\mathrm{bc}}$ & $2.01^{\mathrm{c}}$ & 100.00 \\
\hline \multirow[t]{2}{*}{$\mathrm{S}$} & Lit room & $52.11^{\mathrm{d}}$ & $132.34^{\mathrm{a}}$ & $2.55^{\mathrm{a}}$ & 100.00 \\
\hline & Dark room & $59.97^{\mathrm{a}}$ & $121.29^{\mathrm{ab}}$ & $2.02^{\mathrm{c}}$ & 100.00 \\
\hline Pooled SEM & & 1.34 & 2.13 & 0.06 & 0.03 \\
\hline \multicolumn{6}{|l|}{ Main effects } \\
\hline \multicolumn{6}{|l|}{ Feed brands } \\
\hline $\mathrm{P}$ & & $56.17^{\mathrm{wx}}$ & $119.15^{x}$ & $2.14^{\mathrm{wx}}$ & 98.75 \\
\hline Q & & $57.73^{w}$ & $119.48^{x}$ & $2.11^{\mathrm{wx}}$ & 97.50 \\
\hline $\mathrm{R}$ & & $54.92^{\mathrm{x}}$ & $105.36^{y}$ & $1.94^{\mathrm{x}}$ & 98.75 \\
\hline $\mathrm{S}$ & & $56.05^{\mathrm{wx}}$ & $126.82^{\mathrm{w}}$ & $2.29^{\mathrm{w}}$ & 100.00 \\
\hline \multicolumn{6}{|c|}{ Storage condition } \\
\hline Lit room & & $54.74^{\mathrm{n}}$ & $117.90^{\mathrm{m}}$ & $2.17^{\mathrm{m}}$ & 98.12 \\
\hline Dark room & & $57.69^{\mathrm{m}}$ & $117.61^{\mathrm{n}}$ & $2.06^{\mathrm{n}}$ & 99.37 \\
\hline \multicolumn{6}{|c|}{ Probability (P-Value) } \\
\hline Interaction & & 0.003 & 0.003 & 0.015 & 0.651 \\
\hline Feed brands & & 0.009 & 0.004 & 0.020 & 0.818 \\
\hline Storage condit & & 0.004 & 0.002 & 0.001 & 0.794 \\
\hline
\end{tabular}

Nutrient retention as influenced by main and interaction effects of storage conditions and feed brands are shown in Table 3. Broilers fed feed P stored in dark room had higher $(\mathrm{P} \leq 0.010)$ dry matter, crude fat and fibre retention. However, significantly $(\mathrm{P}<0.001)$ higher crude protein and ash retention were recorded in broilers fed feed $\mathrm{Q}$ and $\mathrm{S}$ stored in dark room, respectively. Higher $(\mathrm{P}<0.05)$ dry matter and crude fat retention were observed in broilers fed feed $\mathrm{P}$ while those fed feed $\mathrm{Q}$ and $\mathrm{S}$ had higher $(\mathrm{P}<0.01)$ crude protein and ash, respectively. Broiler chickens fed feeds stored in dark room had $(\mathrm{P}<0.05)$ higher crude fat $(2.97 \%)$, protein $(2.36 \%)$, fibre $(2.43 \%)$ and ash retention $(3.89 \%)$ when compared to their counterparts fed feeds stored in lit room.

The main and interaction effects of feed brands and storage conditions on haematology of broiler chickens are presented in Table 4. Broilers fed feed $\mathrm{S}$ stored in lit room had lower $(\mathrm{P}<0.01) \mathrm{PCV}$ and RBC. Higher $(\mathrm{P}<0.01)$ WBC was recorded in broilers fed feed $\mathrm{Q}$ stored in dark room compared to other groups. Meanwhile, broilers fed feed S stored in 


\section{Fafiolu and Alabi}

Table 3: Nutrient retention (\%) of broiler chickens fed stored finished feeds

\begin{tabular}{|c|c|c|c|c|c|c|}
\hline Feed brands & $\begin{array}{l}\text { Storage } \\
\text { condition }\end{array}$ & Dry matter & Crude fat & Crude protein & $\begin{array}{l}\text { Crude } \\
\text { fibre }\end{array}$ & Crude ash \\
\hline \multirow[t]{2}{*}{$\mathrm{P}$} & Lit room & $62.42^{\mathrm{ab}}$ & $83.17^{\mathrm{bc}}$ & $72.77^{\mathrm{d}}$ & $61.79^{\text {cde }}$ & $52.23^{\mathrm{d}}$ \\
\hline & Dark room & $64.31^{\mathrm{a}}$ & $86.17^{\mathrm{a}}$ & $74.72^{b}$ & $63.96^{\mathrm{a}}$ & $56.53^{\mathrm{bc}}$ \\
\hline \multirow[t]{2}{*}{ Q } & Lit room & $61.78^{\mathrm{b}}$ & $78.83^{\mathrm{d}}$ & $74.67^{b}$ & $61.15^{\mathrm{de}}$ & $56.70^{\mathrm{bc}}$ \\
\hline & Dark room & $61.33^{\mathrm{b}}$ & $80.68^{\mathrm{cd}}$ & $77.89^{\mathrm{a}}$ & $62.37^{\mathrm{bc}}$ & $58.58^{\mathrm{ab}}$ \\
\hline \multirow[t]{2}{*}{$\mathrm{R}$} & Lit room & $61.97^{\mathrm{b}}$ & $78.81^{\mathrm{d}}$ & $72.04^{\mathrm{d}}$ & $60.96^{\mathrm{e}}$ & $55.67^{\mathrm{c}}$ \\
\hline & Dark room & $63.19^{\mathrm{ab}}$ & $81.90^{\mathrm{bc}}$ & $73.14^{\mathrm{cd}}$ & $62.18^{\mathrm{bcd}}$ & $55.92^{\mathrm{c}}$ \\
\hline \multirow[t]{2}{*}{$\mathrm{S}$} & Lit room & $62.57^{\mathrm{ab}}$ & $81.92^{\mathrm{bc}}$ & $74.10^{\mathrm{bc}}$ & $61.70^{\text {cde }}$ & $58.33^{\mathrm{b}}$ \\
\hline & Dark room & $62.75^{\mathrm{ab}}$ & $83.58^{\mathrm{ab}}$ & $74.74^{b}$ & $63.03^{\mathrm{ab}}$ & $60.58^{\mathrm{a}}$ \\
\hline Pooled SEM & & 0.23 & 0.52 & 0.36 & 0.20 & 0.50 \\
\hline \multicolumn{7}{|l|}{ Main effects } \\
\hline \multicolumn{7}{|l|}{ Feed brands } \\
\hline$P$ & & $63.37^{\mathrm{w}}$ & $84.67^{\mathrm{w}}$ & $73.74^{x}$ & 62.87 & $54.38^{\mathrm{y}}$ \\
\hline Q & & $61.56^{x}$ & $79.76^{y}$ & $76.28^{w}$ & 61.76 & $57.64^{\mathrm{wx}}$ \\
\hline $\mathrm{R}$ & & $62.58^{\mathrm{wx}}$ & $80.35^{x y}$ & $72.59^{\mathrm{x}}$ & 61.57 & $55.79^{x y}$ \\
\hline $\mathrm{S}$ & & $62.66^{\mathrm{wx}}$ & $82.75^{\mathrm{wx}}$ & $74.42^{\mathrm{wx}}$ & 62.37 & $59.46^{\mathrm{w}}$ \\
\hline \multicolumn{7}{|c|}{ Storage condition } \\
\hline Lit room & & 62.18 & $80.68^{\mathrm{n}}$ & $73.39^{n}$ & $61.40^{\mathrm{n}}$ & $55.73^{\mathrm{n}}$ \\
\hline Dark room & & 62.90 & $83.08^{\mathrm{m}}$ & $75.12^{\mathrm{m}}$ & $62.89^{\mathrm{m}}$ & $57.90^{\mathrm{m}}$ \\
\hline \multicolumn{7}{|c|}{ Probability (P-Value) } \\
\hline Interaction & & 0.010 & $<0.001$ & $<0.001$ & $<0.001$ & $<0.001$ \\
\hline Feed brands & & 0.029 & $<0.001$ & $<0.001$ & 0.083 & $<0.001$ \\
\hline Storage condi & & 0.116 & 0.016 & 0.011 & $<0.001$ & 0.027 \\
\hline
\end{tabular}

Table 4: Haematology indices of broiler chickens as influenced by feeding stored finished feeds

\begin{tabular}{|c|c|c|c|c|c|c|c|c|}
\hline $\begin{array}{l}\text { Feed } \\
\text { brands }\end{array}$ & $\begin{array}{l}\text { Storage } \\
\text { condition }\end{array}$ & $\begin{array}{l}\text { PCV } \\
(\%)\end{array}$ & $\begin{array}{c}\mathrm{RBC} \\
\left(\mathrm{x} 10^{12} / \mathrm{L}\right)\end{array}$ & $\begin{array}{c}\text { WBC } \\
\left(\times 10^{9} / \mathrm{L}\right)\end{array}$ & $\begin{array}{c}\text { Neut }^{1} \\
(\%)\end{array}$ & $\begin{array}{c}\text { Lymp }^{2} \\
(\%)\end{array}$ & $\begin{array}{l}\text { Mon }^{3} \\
(\%)\end{array}$ & $\begin{array}{l}\text { Eos }^{4} \\
(\%)\end{array}$ \\
\hline \multirow[t]{2}{*}{$\mathrm{P}$} & Lit room & $37.38^{\mathrm{b}}$ & $5.16^{\mathrm{abc}}$ & $4.57^{\mathrm{c}}$ & $35.38^{\mathrm{ab}}$ & $60.75^{\mathrm{ab}}$ & $2.75^{\mathrm{ab}}$ & 1.50 \\
\hline & Dark room & $38.75^{\mathrm{b}}$ & $5.90^{\mathrm{abc}}$ & $5.85^{\mathrm{bc}}$ & $34.38^{\mathrm{ab}}$ & $62.13^{\mathrm{a}}$ & $2.25^{\mathrm{abc}}$ & 1.00 \\
\hline \multirow[t]{2}{*}{ Q } & Lit room & $36.50^{\mathrm{b}}$ & $4.72^{b c}$ & $3.22^{\mathrm{d}}$ & $38.00^{\mathrm{ab}}$ & $60.00^{\mathrm{b}}$ & $1.00^{\mathrm{bc}}$ & 1.00 \\
\hline & Dark room & $37.50^{b}$ & $5.43^{\mathrm{abc}}$ & $9.95^{\mathrm{a}}$ & $28.00^{\mathrm{b}}$ & $69.00^{\mathrm{a}}$ & $3.00^{\mathrm{ab}}$ & 0.00 \\
\hline \multirow[t]{2}{*}{$\mathrm{R}$} & Lit room & $38.00^{\mathrm{b}}$ & $5.55^{\mathrm{abc}}$ & $4.95^{\mathrm{c}}$ & $31.00^{\mathrm{b}}$ & $65.50^{\mathrm{a}}$ & $3.00^{\mathrm{ab}}$ & 2.00 \\
\hline & Dark room & $40.50^{\mathrm{a}}$ & $6.77^{\mathrm{ab}}$ & $6.56^{\mathrm{b}}$ & $32.50^{\mathrm{b}}$ & $61.50^{\mathrm{ab}}$ & $3.50^{\mathrm{ab}}$ & 1.00 \\
\hline \multirow[t]{2}{*}{ S } & Lit room & $27.50^{c}$ & $3.84^{\mathrm{c}}$ & $5.30^{\mathrm{bc}}$ & $29.50^{\mathrm{b}}$ & $65.50^{\mathrm{a}}$ & $4.00^{\mathrm{a}}$ & 1.00 \\
\hline & Dark room & $43.00^{\mathrm{a}}$ & $7.00^{\mathrm{a}}$ & $2.70^{\mathrm{d}}$ & $46.00^{\mathrm{a}}$ & $52.00^{c}$ & $0.00^{c}$ & 2.00 \\
\hline \multicolumn{2}{|c|}{ Pooled SEM } & 1.00 & 0.24 & 0.50 & 1.33 & 1.16 & 0.30 & 0.18 \\
\hline \multicolumn{9}{|c|}{ Main effects } \\
\hline \multicolumn{9}{|c|}{ Feed brands } \\
\hline \multicolumn{2}{|l|}{$\mathrm{P}$} & $38.06^{\mathrm{wx}}$ & $5.53^{\mathrm{wx}}$ & $5.21^{\mathrm{x}}$ & $34.88^{\mathrm{wx}}$ & $61.44^{x}$ & 2.50 & 1.25 \\
\hline \multicolumn{2}{|l|}{ Q } & $37.00^{\mathrm{y}}$ & $5.08^{x}$ & $6.59^{\mathrm{w}}$ & $33.00^{\mathrm{wx}}$ & $64.50^{\mathrm{w}}$ & 2.00 & 0.50 \\
\hline \multicolumn{2}{|l|}{ R } & $39.25^{\mathrm{w}}$ & $6.16^{\mathrm{w}}$ & $5.76^{\mathrm{x}}$ & $31.75^{\mathrm{x}}$ & $63.50^{\mathrm{w}}$ & 3.25 & 1.50 \\
\hline \multirow{2}{*}{\multicolumn{2}{|c|}{$\begin{array}{l}\text { S } \\
\text { Storage condition }\end{array}$}} & $35.25^{\mathrm{y}}$ & $5.42^{\mathrm{wx}}$ & $4.00^{y}$ & $37.75^{\mathrm{w}}$ & $58.75^{\mathrm{y}}$ & 2.00 & 1.50 \\
\hline & & & & & & & & \\
\hline \multicolumn{2}{|c|}{ Lit room } & $34.84^{\mathrm{n}}$ & $4.82^{\mathrm{n}}$ & $4.51^{\mathrm{n}}$ & $33.47^{\mathrm{n}}$ & $62.94^{\mathrm{m}}$ & 2.69 & 1.38 \\
\hline \multicolumn{2}{|c|}{ Dark room } & $39.94^{\mathrm{m}}$ & $6.27^{\mathrm{m}}$ & $6.26^{\mathrm{m}}$ & $35.22^{\mathrm{m}}$ & $61.16^{\mathrm{n}}$ & 2.19 & 1.00 \\
\hline \multicolumn{9}{|c|}{ Probability (P-Value) } \\
\hline \multicolumn{2}{|c|}{ Interaction } & $<0.001$ & 0.002 & 0.001 & 0.003 & 0.001 & 0.001 & 0.073 \\
\hline \multicolumn{2}{|c|}{ Feed brands } & 0.036 & 0.004 & 0.010 & 0.023 & 0.014 & 0.430 & 0.148 \\
\hline \multicolumn{2}{|c|}{ Storage condition } & 0.008 & 0.007 & 0.001 & 0.003 & 0.003 & 0.417 & 0.303 \\
\hline
\end{tabular}

${ }_{\mathrm{a}, \mathrm{b}, \mathrm{b}, \mathrm{d}, \mathrm{e}}$ Interaction, ${ }^{\mathrm{w}, \mathrm{x}, \mathrm{y}}$ Feed brands, ${ }^{\mathrm{m}, \mathrm{n}}$ Storage condition - means in columns bearing different superscript differ significantly $(\mathrm{P}<0.05)$. $\quad{ }^{1}$ Neut - Neutrophils; ${ }^{2}$ Lymp - Lymphocytes; ${ }^{3}$ Mon Monocytes; ${ }^{4}$ Eos - Eosinophils. 


\section{Diet matrix and broiler chicken performance}

dark room recorded higher $(\mathrm{P}<0.05)$ neutrophils and lower lymphocytes when compared with other treatments. Significantly $(\mathrm{P}<0.01)$ higher monocyte was recorded in broilers fed feed $\mathrm{S}$ stored in lit room. Regarding main effects, higher $(\mathrm{P}<0.05) \mathrm{PCV}$ and $\mathrm{RBC}$ were observed in broilers fed feed $\mathrm{R}$ while those fed feed $\mathrm{Q}$ and $\mathrm{S}$ had higher WBC and neutrophil, respectively. Chickens fed feeds stored in dark room had $(\mathrm{P}<0.05)$ higher $\mathrm{PCV}$ (14.64\%), RBC (30.08\%), WBC (38.80\%), neutrophils $(5.23 \%)$ and reduced lymphocytes $(2.83 \%)$ compared to their counterparts fed feeds stored in lit room. Influence of feed brands and storage conditions interaction significantly $(\mathrm{P} \leq$ 0.003 ) altered the serum biochemical indices (except AST; $\mathrm{P}=0.107$ ) of broiler chickens (Table 5). Lower total protein $(51.50 \mathrm{~g} / \mathrm{L})$ and globulin $(20.15 \mathrm{~g} / \mathrm{L})$ were recorded in broilers fed feed Q stored in dark room compared to other groups. Higher uric acid and creatinine concentration were recorded in broilers fed feed $\mathrm{R}$ and $\mathrm{S}$ stored in lit room, respectively. Among feed brands, chickens fed with feed $\mathrm{R}$ had higher $(\mathrm{P}<0.001)$ total protein, albumin, Albumin/globulin ratio, and uric acid while those fed feed $\mathrm{S}$ had higher $(\mathrm{P}=0.032)$ globulin values. Broilers fed feeds stored in lit room had higher $(\mathrm{P}<0.05)$ globulin $(9.7 \%)$, uric acid (13.6\%), AST (15.2\%) and ALT (47.6\%) when compared with those fed feeds stored in dark room.

Table 5: Effect of feeding stored finished feeds on serum biochemical indices of broiler chickens

\begin{tabular}{|c|c|c|c|c|c|c|c|c|c|}
\hline $\begin{array}{l}\text { Feed } \\
\text { brands }\end{array}$ & $\begin{array}{l}\text { Storage } \\
\text { condition }\end{array}$ & $\begin{array}{l}\text { T. } \\
\text { Prot. }{ }^{1} \\
\text { (g/L) }\end{array}$ & $\begin{array}{l}\text { Albumin } \\
(\mathrm{g} / \mathrm{L})\end{array}$ & $\begin{array}{l}\text { Globuli } \\
\text { n (g/L) }\end{array}$ & $\mathrm{A}: \mathrm{G}^{2}$ & $\begin{array}{c}\text { Uric } \\
\text { acid } \\
(\mathrm{mg} / \mathrm{dL})\end{array}$ & $\begin{array}{c}\text { Creat }^{3} \\
(\mathrm{mg} / \mathrm{dL} \\
)\end{array}$ & $\begin{array}{c}\text { AST } \\
(\mathrm{IU} / \mathrm{L})\end{array}$ & $\begin{array}{c}\text { ALT } \\
(\mathrm{IU} / \mathrm{L})\end{array}$ \\
\hline \multirow[t]{2}{*}{$\mathrm{P}$} & Lit room & $58.93^{\mathrm{ab}}$ & $33.31^{\mathrm{b}}$ & $25.60^{\mathrm{a}}$ & $1.39^{\mathrm{bc}}$ & $4.46^{\mathrm{b}}$ & $1.40^{\mathrm{ab}}$ & 144.01 & $\underset{b}{41.94^{\mathrm{a}}}$ \\
\hline & $\begin{array}{l}\text { Dark } \\
\text { room }\end{array}$ & $57.90^{\mathrm{ab}}$ & $33.35^{\mathrm{b}}$ & $24.55^{\mathrm{ab}}$ & $1.39^{\mathrm{bc}}$ & $4.04^{\mathrm{bcd}}$ & $1.21^{\mathrm{b}}$ & 123.44 & $28.39^{b}$ \\
\hline \multirow[t]{2}{*}{ Q } & Lit room & $57.20^{\mathrm{b}}$ & $30.50^{\mathrm{c}}$ & $26.65^{\mathrm{a}}$ & $1.15^{\mathrm{d}}$ & $3.95^{\text {bcd }}$ & $1.20^{\mathrm{b}}$ & 153.35 & $36.85^{\mathrm{b}}$ \\
\hline & $\begin{array}{l}\text { Dark } \\
\text { room }\end{array}$ & $51.50^{c}$ & $31.35^{\mathrm{bc}}$ & $20.15^{\mathrm{c}}$ & $1.56^{\mathrm{ab}}$ & $3.45^{\mathrm{d}}$ & $1.25^{\mathrm{b}}$ & 132.45 & $23.30^{\mathrm{b}}$ \\
\hline \multirow[t]{2}{*}{$\mathrm{R}$} & Lit room & $61.10^{\mathrm{a}}$ & $37.05^{\mathrm{a}}$ & $24.05^{\mathrm{ab}}$ & $1.54^{\mathrm{ab}}$ & $5.15^{\mathrm{a}}$ & $1.15^{\mathrm{b}}$ & 140.15 & $67.60^{\mathrm{a}}$ \\
\hline & $\begin{array}{l}\text { Dark } \\
\text { room }\end{array}$ & $61.30^{\mathrm{a}}$ & $38.75^{\mathrm{a}}$ & $22.55^{\mathrm{bc}}$ & $1.72^{\mathrm{a}}$ & $4.40^{\mathrm{b}}$ & $1.45^{\mathrm{ab}}$ & 111.75 & $30.90^{\mathrm{b}}$ \\
\hline \multirow[t]{2}{*}{$\mathrm{S}$} & Lit room & $58.30^{\mathrm{ab}}$ & $31.55^{b c}$ & $26.75^{\mathrm{a}}$ & $1.19^{\mathrm{cd}}$ & $4.15^{\mathrm{bc}}$ & $1.65^{\mathrm{a}}$ & 134.55 & $22.00^{\mathrm{b}}$ \\
\hline & $\begin{array}{l}\text { Dark } \\
\text { room }\end{array}$ & $56.60^{\mathrm{b}}$ & $29.95^{\mathrm{c}}$ & $26.65^{\mathrm{a}}$ & $1.13^{\mathrm{d}}$ & $3.70^{\mathrm{cd}}$ & $1.10^{\mathrm{b}}$ & 128.95 & $31.50^{\mathrm{b}}$ \\
\hline Pooled & EM & 0.64 & 0.63 & 0.49 & 0.04 & 0.11 & 0.04 & 3.62 & 3.26 \\
\hline \multicolumn{10}{|c|}{ Feed brands } \\
\hline \multicolumn{2}{|c|}{ P } & $58.41^{\mathrm{wx}}$ & $33.33^{\mathrm{x}}$ & $25.08^{\mathrm{wx}}$ & $1.39^{x}$ & $4.25^{\mathrm{wx}}$ & 1.31 & 133.73 & 35.16 \\
\hline \multicolumn{2}{|l|}{ Q } & $54.35^{\mathrm{y}}$ & $30.93^{y}$ & $23.40^{x}$ & $1.35^{\mathrm{xy}}$ & $3.70^{\mathrm{x}}$ & 1.23 & 142.90 & 30.08 \\
\hline \multicolumn{2}{|l|}{$\mathrm{R}$} & $61.20^{\mathrm{w}}$ & $37.90^{\mathrm{w}}$ & $23.30^{\mathrm{x}}$ & $1.63^{\mathrm{w}}$ & $4.78^{w}$ & 1.30 & 125.95 & 49.25 \\
\hline \multicolumn{2}{|l|}{$\mathrm{S}$} & $57.45^{x y}$ & $30.75^{y}$ & $26.70^{\mathrm{w}}$ & $1.16^{\mathrm{y}}$ & $3.93^{\mathrm{x}}$ & 1.38 & 131.75 & 26.75 \\
\hline \multicolumn{10}{|c|}{ Storage condition } \\
\hline \multicolumn{2}{|l|}{$\begin{array}{l}\text { Lit } \\
\text { room }\end{array}$} & 58.88 & 33.10 & $25.76^{\mathrm{m}}$ & 1.32 & $4.43^{\mathrm{m}}$ & 1.35 & $\underset{\mathrm{m}}{143.02}$ & $\underset{\mathrm{m}}{42.10}$ \\
\hline \multicolumn{2}{|c|}{ Dark room } & 56.83 & 33.35 & $23.48^{n}$ & 1.45 & $3.90^{\mathrm{n}}$ & 1.25 & $124.15^{\mathrm{n}}$ & $28.52^{n}$ \\
\hline \multicolumn{10}{|c|}{ Probability (P-Value) } \\
\hline \multicolumn{2}{|c|}{ Interaction } & $<0.001$ & $<0.001$ & $<0.001$ & $<0.001$ & $<0.001$ & 0.003 & 0.107 & 0.001 \\
\hline \multicolumn{2}{|c|}{ Feed brands } & $<0.001$ & $<0.001$ & 0.032 & $<0.001$ & $<0.001$ & 0.703 & 0.441 & 0.031 \\
\hline \multicolumn{2}{|c|}{ Storage condition } & 0.107 & 0.851 & 0.016 & 0.129 & 0.012 & 0.268 & 0.006 & 0.034 \\
\hline
\end{tabular}




\section{Fafiolu and Alabi}

Effects of feeding stored finished feeds on intestinal morphology of finishing broiler chickens are presented in Table 6. Broilers fed feed $\mathrm{R}$ stored in dark room had higher $(\mathrm{P}<0.001)$ duodenal villus height $(\mathrm{VH})$, villus height/crypt depth ratio (VCR), and reduced luminal propria depth (LPD). At the jejunum, broilers fed feed Q stored in dark room had higher $(\mathrm{P} \leq 0.002) \mathrm{VH}, \mathrm{VCR}$ and LPD whereas higher ileal $(\mathrm{P}<0.001)$
VH and VCR were observed in broilers fed feed $\mathrm{R}$ stored in dark room. Feed types effect showed that broilers fed with feed Q had higher duodenal LPD $(0.23 \mathrm{~mm})$ and jejunal VH $(0.85 \mathrm{~mm})$, however, broilers fed feed $\mathrm{R}$ had higher $(\mathrm{P}<0.05)$ ileal $\mathrm{VH}$ (0.88 mm), VCR (3.26) and LPD (0.30 $\mathrm{mm})$. Broilers fed feeds stored in dark room had higher $(\mathrm{P}<0.05) \mathrm{VH}$ and VCR at all the intestinal segments.

Table 6: Effect of feeding stored finished feeds on intestinal morphology ( $\mathrm{mm})$ of broiler chickens

\begin{tabular}{|c|c|c|c|c|c|c|c|c|c|c|}
\hline \multirow{2}{*}{$\begin{array}{l}\text { Feed } \\
\text { brands }\end{array}$} & \multirow{2}{*}{$\begin{array}{l}\text { Storage } \\
\text { condition }\end{array}$} & \multicolumn{3}{|c|}{ Duodenum } & \multicolumn{3}{|c|}{ Jejunum } & \multicolumn{3}{|c|}{ Ileum } \\
\hline & & $\mathrm{VH}^{1}$ & $\mathrm{LPD}^{2}$ & $\mathrm{VCR}^{3}$ & $\mathrm{VH}$ & LPD & VCR & $\mathrm{VH}$ & LPD & VCR \\
\hline \multirow[t]{2}{*}{$\mathrm{P}$} & Lit room & $1.24^{\mathrm{d}}$ & $0.21^{\mathrm{ab}}$ & $5.88^{b}$ & $0.61^{\mathrm{bc}}$ & $0.20^{\mathrm{ab}}$ & $3.01^{\mathrm{bc}}$ & $0.62^{\mathrm{d}}$ & $0.28^{b}$ & $2.21^{\mathrm{de}}$ \\
\hline & $\begin{array}{l}\text { Dark } \\
\text { room }\end{array}$ & $1.27^{\mathrm{c}}$ & $0.19^{\mathrm{bc}}$ & $6.52^{b}$ & $0.98^{\mathrm{ab}}$ & $0.19^{\mathrm{ab}}$ & $5.16^{\mathrm{ab}}$ & $0.73^{c}$ & $0.27^{b}$ & $2.67^{\mathrm{cd}}$ \\
\hline \multirow[t]{2}{*}{ Q } & Lit room & $0.95^{\mathrm{g}}$ & $0.23^{\mathrm{a}}$ & $4.13^{\mathrm{d}}$ & $0.45^{\mathrm{c}}$ & $0.18^{\mathrm{bc}}$ & $2.50^{\mathrm{c}}$ & $0.49^{\mathrm{e}}$ & $0.23^{\mathrm{cd}}$ & $2.13^{\mathrm{e}}$ \\
\hline & $\begin{array}{l}\text { Dark } \\
\text { room }\end{array}$ & $1.40^{\mathrm{b}}$ & $0.23^{\mathrm{a}}$ & $6.09^{b}$ & $1.25^{\mathrm{a}}$ & $0.21^{\mathrm{a}}$ & $5.96^{\mathrm{a}}$ & $0.50^{\mathrm{e}}$ & $0.20^{\mathrm{d}}$ & $2.50^{\mathrm{de}}$ \\
\hline \multirow[t]{2}{*}{$\mathrm{R}$} & Lit room & $1.00^{\mathrm{f}}$ & $0.20^{\mathrm{bc}}$ & $5.03^{c}$ & $0.65^{\mathrm{bc}}$ & $0.20^{\mathrm{ab}}$ & $3.27^{b c}$ & $0.86^{\mathrm{b}}$ & $0.40^{\mathrm{a}}$ & $2.15^{\mathrm{e}}$ \\
\hline & $\begin{array}{l}\text { Dark } \\
\text { room }\end{array}$ & $1.71^{\mathrm{a}}$ & $0.18^{c}$ & $9.52^{\mathrm{a}}$ & $0.70^{\mathrm{bc}}$ & $0.17^{\mathrm{c}}$ & $4.12^{\mathrm{abc}}$ & $0.90^{\mathrm{a}}$ & $0.21^{\mathrm{d}}$ & $4.37^{\mathrm{a}}$ \\
\hline \multirow[t]{2}{*}{$\mathrm{S}$} & Lit room & $1.10^{\mathrm{e}}$ & $0.20^{\mathrm{bc}}$ & $5.79^{b}$ & $0.53^{\mathrm{c}}$ & $0.17^{\mathrm{c}}$ & $3.19^{\mathrm{bc}}$ & $0.60^{\mathrm{d}}$ & $0.20^{\mathrm{d}}$ & $3.02^{\mathrm{bc}}$ \\
\hline & $\begin{array}{l}\text { Dark } \\
\text { room }\end{array}$ & $1.00^{\mathrm{f}}$ & $0.21^{\mathrm{ab}}$ & $4.77^{\mathrm{cd}}$ & $0.98^{\mathrm{ab}}$ & $0.20^{\mathrm{ab}}$ & $4.91^{\mathrm{abc}}$ & $0.83^{\mathrm{b}}$ & $0.25^{\mathrm{bc}}$ & $3.32^{\mathrm{b}}$ \\
\hline \multicolumn{2}{|c|}{ Pooled SEM } & 49.93 & 3.84 & 0.32 & 58.42 & 3.38 & 0.28 & 31.64 & 13.12 & 0.15 \\
\hline \multicolumn{11}{|c|}{ Main effects } \\
\hline \multicolumn{11}{|c|}{ Feed brands } \\
\hline $\mathrm{P}$ & & 1.25 & $0.20^{\mathrm{x}}$ & 6.20 & $0.80^{\mathrm{w}}$ & 0.20 & 4.09 & $0.68^{x}$ & $0.28^{x}$ & $2.44^{\mathrm{x}}$ \\
\hline Q & & 1.18 & $0.23^{\mathrm{w}}$ & 5.11 & $0.85^{\mathrm{w}}$ & 0.20 & 4.23 & $0.50^{y}$ & $0.22^{y}$ & $2.32^{x}$ \\
\hline $\mathrm{R}$ & & 1.36 & $0.19^{x}$ & 7.27 & $0.68^{y}$ & 0.19 & 3.69 & $0.88^{w}$ & $0.30^{\mathrm{w}}$ & $3.26^{\mathrm{w}}$ \\
\hline $\mathrm{S}$ & & 1.05 & $0.20^{\mathrm{x}}$ & 5.28 & $0.75^{\mathrm{x}}$ & 0.19 & 4.05 & $0.72^{x}$ & $0.23^{\mathrm{y}}$ & $3.17^{\mathrm{w}}$ \\
\hline \multicolumn{11}{|c|}{ Storage condition } \\
\hline \multicolumn{2}{|c|}{ Lit room } & $1.07^{\mathrm{n}}$ & 0.21 & $5.21^{\mathrm{n}}$ & $0.56^{\mathrm{n}}$ & 0.19 & $2.99^{n}$ & 0.64 & 0.28 & $2.38^{n}$ \\
\hline \multicolumn{2}{|c|}{ Dark room } & $1.35^{\mathrm{m}}$ & 0.20 & $6.72^{\mathrm{m}}$ & $0.98^{\mathrm{m}}$ & 0.19 & $5.04^{\mathrm{m}}$ & 0.74 & 0.23 & $3.22^{\mathrm{m}}$ \\
\hline \multicolumn{11}{|c|}{ Probability (P-Value) } \\
\hline \multicolumn{2}{|c|}{ Interaction } & $<0.001$ & $<0.001$ & $<0.001$ & $<0.001$ & $<0.001$ & 0.002 & $<0.001$ & $<0.001$ & $<0.001$ \\
\hline \multicolumn{2}{|c|}{ Feed brands } & 0.172 & $<0.001$ & 0.054 & 0.029 & 0.504 & 0.930 & $<0.001$ & 0.039 & 0.041 \\
\hline \multicolumn{2}{|c|}{ Storage condition } & 0.004 & 0.637 & 0.015 & $<0.001$ & 0.530 & $<0.001$ & 0.126 & 0.086 & 0.003 \\
\hline
\end{tabular}

Gut microflora population differed significantly $(\mathrm{P}<0.001)$ by feed brands and storage conditions interaction (Table 7). Broilers fed feed Q stored in dark room had higher $(\mathrm{P}<0.001)$ total bacteria and coliforms counts. Higher $(\mathrm{P}<0.001)$ salmonella counts were observed in broilers fed feed $\mathrm{R}$ stored in lit room. Higher $(\mathrm{P}<0.001)$ lactobacillus and lower clostridium counts were recorded in broilers fed feed S and Q stored in lit room, respectively. With respect to feed brands 
Diet matrix and broiler chicken performance

Table 7: Influence of feeding stored finished feeds on gut microbiology $\left(x_{10}^{6} \mathrm{cfu} / \mathrm{mL}\right)$ of broiler chickens

\begin{tabular}{|c|c|c|c|c|c|c|}
\hline $\begin{array}{l}\text { Feed } \\
\text { brands }\end{array}$ & $\begin{array}{l}\text { Storage } \\
\text { condition }\end{array}$ & $\begin{array}{c}\text { Total } \\
\text { bacteria }\end{array}$ & Coliforms & Salmonella & Lactobacillus & Clostridium \\
\hline \multirow[t]{2}{*}{$\mathrm{P}$} & Lit room & $3.05^{\mathrm{d}}$ & $1.71^{\mathrm{d}}$ & $0.13^{\mathrm{e}}$ & $0.77^{\mathrm{d}}$ & $0.68^{c}$ \\
\hline & Dark room & $3.45^{\mathrm{c}}$ & $1.85^{\mathrm{c}}$ & $0.18^{d}$ & $0.77^{\mathrm{d}}$ & $0.94^{\mathrm{b}}$ \\
\hline \multirow[t]{2}{*}{ Q } & Lit room & $3.51^{\mathrm{c}}$ & $1.90^{\mathrm{c}}$ & $0.00^{f}$ & $0.45^{\mathrm{e}}$ & $0.25^{\mathrm{e}}$ \\
\hline & Dark room & $5.46^{\mathrm{a}}$ & $2.65^{\mathrm{a}}$ & $0.30^{\mathrm{c}}$ & $1.20^{\mathrm{b}}$ & $0.70^{\mathrm{c}}$ \\
\hline \multirow[t]{2}{*}{$\mathrm{R}$} & Lit room & $2.35^{\mathrm{e}}$ & $1.60^{\mathrm{e}}$ & $0.50^{\mathrm{a}}$ & $0.85^{\mathrm{d}}$ & $0.40^{\mathrm{d}}$ \\
\hline & Dark room & $1.75^{\mathrm{f}}$ & $1.40^{\mathrm{f}}$ & $0.40^{\mathrm{b}}$ & $0.95^{\mathrm{c}}$ & $0.65^{\mathrm{c}}$ \\
\hline \multirow[t]{2}{*}{$\mathrm{S}$} & Lit room & $4.40^{\mathrm{b}}$ & $2.10^{\mathrm{b}}$ & $0.00^{f}$ & $1.30^{\mathrm{a}}$ & $1.45^{\mathrm{a}}$ \\
\hline & Dark room & $4.40^{\mathrm{b}}$ & $1.80^{\mathrm{cd}}$ & $0.00^{f}$ & $0.51^{\mathrm{e}}$ & $1.50^{\mathrm{a}}$ \\
\hline \multicolumn{2}{|c|}{ Pooled SEM } & 0.23 & 0.07 & 0.04 & 0.06 & 0.09 \\
\hline \multicolumn{7}{|c|}{ Main effects } \\
\hline \multicolumn{7}{|c|}{ Feed brands } \\
\hline \multicolumn{2}{|l|}{$\mathrm{P}$} & $3.25^{\mathrm{x}}$ & $1.78^{x y}$ & $0.15^{x}$ & 0.77 & $0.81^{\mathrm{x}}$ \\
\hline \multicolumn{2}{|l|}{ Q } & $4.49^{w}$ & $2.28^{\mathrm{w}}$ & $0.15^{\mathrm{x}}$ & 0.83 & $0.48^{y}$ \\
\hline \multicolumn{2}{|l|}{$\mathrm{R}$} & $2.05^{\mathrm{y}}$ & $1.50^{\mathrm{y}}$ & $0.45^{\mathrm{w}}$ & 0.90 & $0.53^{\mathrm{y}}$ \\
\hline \multicolumn{2}{|l|}{ S } & $4.40^{\mathrm{w}}$ & $1.95^{\mathrm{wx}}$ & $0.00^{\mathrm{y}}$ & 0.91 & $1.48^{\mathrm{w}}$ \\
\hline \multicolumn{7}{|c|}{ Storage condition } \\
\hline \multicolumn{2}{|c|}{ Lit room } & 3.33 & 1.83 & 0.16 & 0.84 & 0.70 \\
\hline \multicolumn{2}{|c|}{ Dark room } & 3.77 & 1.93 & 0.22 & 0.86 & 0.95 \\
\hline \multicolumn{7}{|c|}{ Probability (P-Value) } \\
\hline \multicolumn{2}{|c|}{ Interaction } & $<0.001$ & $<0.001$ & $<0.001$ & $<0.001$ & $<0.001$ \\
\hline \multicolumn{2}{|c|}{ Feed brands } & $<0.001$ & $<0.001$ & $<0.001$ & 0.832 & $<0.001$ \\
\hline \multicolumn{2}{|c|}{ Storage condition } & 0.360 & 0.521 & 0.425 & 0.913 & 0.157 \\
\hline
\end{tabular}

Table 8: Influence of feeding stored finished feeds on carcass quality traits (\% live weight) of broiler chickens

\begin{tabular}{|c|c|c|c|c|c|c|}
\hline Feed brands & $\begin{array}{l}\text { Storage } \\
\text { condition }\end{array}$ & $\begin{array}{c}\text { Dressed } \\
\text { yield }\end{array}$ & Breast & Drumstick & Thigh & $\begin{array}{c}\text { Empty } \\
\text { Gizzard }\end{array}$ \\
\hline \multirow[t]{2}{*}{$\mathrm{P}$} & Lit room & $72.17^{\mathrm{ab}}$ & 18.51 & 9.93 & 9.54 & 1.94 \\
\hline & Dark room & $69.77^{b}$ & 19.23 & 8.95 & 10.28 & 1.68 \\
\hline \multirow[t]{2}{*}{ Q } & Lit room & $67.21^{\mathrm{b}}$ & 17.57 & 9.77 & 9.13 & 1.97 \\
\hline & Dark room & $67.65^{\mathrm{b}}$ & 19.31 & 9.76 & 9.80 & 1.75 \\
\hline \multirow[t]{2}{*}{$\mathrm{R}$} & Lit room & $73.02^{\mathrm{ab}}$ & 19.87 & 9.94 & 9.37 & 1.82 \\
\hline & Dark room & $67.12^{\mathrm{b}}$ & 17.99 & 9.82 & 9.91 & 1.74 \\
\hline \multirow[t]{2}{*}{$\mathrm{S}$} & Lit room & $76.42^{\mathrm{a}}$ & 19.22 & 10.12 & 10.32 & 2.09 \\
\hline & Dark room & $74.15^{\mathrm{ab}}$ & 21.39 & 10.15 & 99.92 & 1.71 \\
\hline Pooled SEM & & 1.56 & 0.38 & 0.53 & 0.30 & 0.05 \\
\hline \multicolumn{7}{|l|}{ Main effects } \\
\hline \multicolumn{7}{|l|}{ Feed brands } \\
\hline $\mathrm{P}$ & & 70.97 & 18.87 & 9.44 & 9.91 & 1.81 \\
\hline Q & & 67.43 & 18.44 & 9.77 & 9.46 & 1.86 \\
\hline $\mathrm{R}$ & & 70.07 & 18.93 & 9.88 & 9.64 & 1.78 \\
\hline $\mathrm{S}$ & & 75.29 & 20.30 & 10.14 & 10.12 & 1.90 \\
\hline \multicolumn{7}{|c|}{ Storage condition } \\
\hline Lit room & & $72.21^{\mathrm{m}}$ & 18.79 & $9.94^{\mathrm{m}}$ & 9.59 & 1.95 \\
\hline Dark room & & $69.67^{\mathrm{n}}$ & 19.40 & $9.67^{\mathrm{n}}$ & 9.98 & 1.72 \\
\hline \multicolumn{7}{|c|}{ Probability (P-Value) } \\
\hline Interaction & & 0.008 & 0.302 & 0.524 & 0.986 & 0.453 \\
\hline Feed brands & & 0.121 & 0.355 & 0.922 & 0.892 & 0.853 \\
\hline Storage condi & tion & 0.019 & 0.380 & 0.012 & 0.535 & 0.019 \\
\hline
\end{tabular}




\section{Fafiolu and Alabi}

effect, broiler chickens fed with feed $\mathrm{R}$ had lower $(\mathrm{P}<0.001)$ total bacteria and coliforms counts. No salmonella was isolated but higher clostridium counts were observed in broilers fed feed S. It is worthy of note that storage media had no significant effect $(\mathrm{P}>0.05)$ on the gut microflora population.

Results of carcass traits showed that dressed yield was higher $(\mathrm{P}=0.008)$ in chickens fed feed S stored in lit room while primal cuts such as breast, drumstick, thigh and empty gizzard were not significantly $(\mathrm{P}>0.05)$ affected (Table 8). Regarding main effects, feed brands had no significant $(\mathrm{P}>0.05)$ influence on carcass yield. However, higher dressed yield $(\mathrm{P}=0.019)$ and drumstick $(\mathrm{P}=0.012)$ were recorded in broilers fed feeds stored in lit room.

\section{Discussion}

The critical role of poultry nutrition in the sustainable poultry farming enterprise cannot be over-emphasized. Besides the supply of necessary dietary nutrients for optimal expression of animal's genetic potential and production of qualitative products, efficient utilization of dietary nutrients and cost of feed per weight gain influences the profit margin of poultry farmers (Reddy, 2001). In the current study, feed brands and storage condition influenced the growth performance of growing broiler chickens. One of the possible explanations is the feed matrix (intrinsic nutritive value) of the proprietary feeds used. Finished feed comprises of various ingredients which are properly milled to provide energy and other essential nutrients needed for optimal broiler performance. Often times, variation exists among feeds produced for a class of birds with respect to nutrients basically as a result of the differences in ingredient composition of such diets (Alabi et al., 2017). Since the nutrient contents of broiler finisher feeds assessed are closely similar, the performance of single broiler strain raised under the same management condition is expected to be comparable. However, the production performance is greatly influenced by the quality, in terms of digestibility and bioavailability of nutrients, and not just the nutritional profile of feed offered to broilers (Yegany et al., 2002; Alabi et al., 2018). Muñoz et al. (2018) had noted that the levels of nutritional imbalance in terms of nutrient deficiency or excess, could interfere with feed intake, growth rate and energy expenditure in broiler chickens. The types and inclusion level of additives (such as enzymes, growth-enhancers, immune boosters, toxin binders, antioxidants, etc.) being used for each proprietary feed brand from different company is likely to vary. This gives the compounded feed a physically and nutritionally complex structure, and thus, might have influenced the feed utilization efficiency, body weight gain, immune status, mortality rate, and production economics of the broiler chickens

In addition, better performance in broilers fed diets stored in dark room despite the similarity in temperature and relative humidity of the stores suggests stability and availability of critical nutrients, vitamins and minerals that play vital roles in nutrient utilization and ultimately growth performance. Previous works have established that temperature, relative humidity, light, ventilation, and duration of feed storage could influence feed quality in storage (Reddy, 2001; Alabi et al., 2017). Poor storage could modify the nutritive value and palatability of feed through loss of critical nutrients, enzymatic oxidation, staleness and contamination by insects and microbes. This might result to reduced growth, increased physiologic stress and higher mortality due to poor bird health (Uppal et al., 2004; Waheed et al., 2004; Uchegbu et al., 2009). Poor feed conversion 


\section{Diet matrix and broiler chicken performance}

ratio observed in broilers fed feed $\mathrm{S}$ stored in lit room could be attributed to photostimulation of oxidative rancidity with adverse effect on palatability of feed which could negatively influence the feed consumption and utilization of dietary nutrients for muscle tissue accretion. Nonvariation in livability as mortality recorded across the treatments was less than $3 \%$ agreed with the reports of Lopez et al (2007). In this present study, significant variation in broiler performance agrees with the reports of Orheruata et al. (2006) and Uchegbu et al. (2009) in finisher broilers fed different commercial feed brands. In addition, Tavernari et al. (2014) and Muñoz et al. (2018) observed differences in weight gain and production index of broilers with respect to nutrients levels of the feed and sex.

Digestion of feed and absorption of inherent nutrients are the essential role of gastrointestinal tract (GIT) of broiler chickens. Presence of digestive enzymes, gut microbes and various specialized substances secreted at each distinct region of the GIT help to achieve this great feat. The present data showed that the apparent nutrient digestibility was influenced by both the main and interaction effects. Broilers fed feed $\mathrm{P}$ stored in dark room had higher dry matter, crude fat and fibre retention whereas higher crude protein and ash retention were recorded in broilers fed feed Q and S stored in dark room, respectively. The results in this study differed from those obtained by Lopez et al. (2007), Sakomura and Rostagno (2016) and Muñoz et al. (2018) who reported higher dry matter and lower crude protein digestibility in broilers chickens fed corn-soybean meal based diets. Differences in the digestibility of various feed brands employed in this study are in consonance with the reports of Uchegbu et al. (2009). In contrast, Oyediji et al. (2005) revealed that nutrient retention was not influenced by varying crude protein and feeding regimens of broiler chickens. The discrepancies could be attributed to differences in diet composition, nutrient quality and age of the birds. Broilers fed feeds stored in dark room had significantly higher crude fat, protein, fibre and ash retention. Decrease nutrient digestibility in broilers fed feeds stored in lit room could support the assertion that essential micronutrients required for metabolism might have been lost or unavailable (formed complexes) due to oxidation. More so, increased passage rate of digesta in the presence of stressor could limit digestion. Virden and Kidd (2009) reported that stress could cause significant decline in crude protein digestibility with concomitant polydispsia and polyuria during and after stress. Horn et al. (2010) also reported that reduced nutrient digestibility might come about due to decrease in gut protein synthesis and villi surface area thereby leading to reduced nutrient transport and absorption.

Blood profile analysis is aimed at assessing the role of dietary nutrients on the health status of broiler chickens, and also provides insight into primary physiological functions of some vital organs such as liver and kidney (Adeyemo et al., 2000; Adeleye et al., 2018). Earlier studies have reported several factors which influence the constituents of blood in broiler chickens including age of birds, strain, diet, and management system (Talebi et al., 2005; Fafiolu et al., 2014). Higher PCV and RBC counts were obtained in broilers fed feed $\mathrm{S}$ stored in dark room in this current study. Increased PCV and erythrocytes in broilers would enhance blood cell formation, improve oxygen, $\mathrm{CO}_{2}$ and sugar-carrying capacity of the blood, prevent oxidative damage, and improve health status (Wakenell, 2010; Fafiolu et al., 2014; 2020). WBC counts have been linked with immune response to stress and/or 


\section{Fafiolu and Alabi}

disease-challenge conditions (Fafiolu et al., 2014). Broilers with suppressed immune status would be more susceptible to pathogens and diseases with adverse effects on overall performance (Adeleye et al., 2018). Heterophils, lymphocytes, monocytes and macrophages play crucial roles in defense against invasive pathogens through series of interconnected phagocytic and cytotoxic activities and efforts to secrete cytokines, lymphokines, and specific antibodies needed to conquer individual antigens (Qureshi, 2003; Swaggerty et al., 2003). Chickens fed feed Q stored in dark room recorded higher $\mathrm{WBC}$ and lymphocytes than those fed S stored in dark room. For a chicken to mount an effective immuno-competence against stress or clinical challenge, there would be a paradigm shift in the nutrient utilization towards synthesis of cytokines, immunoglobulins and natural killer cells. This could result in reduced body gain as observed in this study. However, low mortality recorded, absence of clinical symptoms, and non-significance in livability of broiler chickens in this study suggests that the dietary nutrients are capable of supporting the proliferation and maturation of hematopoietic stem cells and immune system functions of the birds.

Serum biochemistry analysis provides insight into the quality of nutrition and various chemical and enzymes that are involved in body's metabolic functions, to detect organs' dysfunction and the overall health status of birds (Fafiolu et al., 2014; Adeleye et al., 2018). It also serves as an important diagnostic tool to detect, diagnose, and monitor various diseases and pathological processes (Tothova et al., 2016). The interaction of feed brands and storage conditions showed signiflcantly higher serum protein, albumin, uric acid and ALT values in broilers fed feed R stored in lit room compared with other groups. Blood total protein level is a pointer to how changes in protein metabolism in the body affect growth rate, health status and meat yield (Adeyemo et al., 2000). Proteins are the major constituents of blood serum and they participate actively in various physiological functions including homeostasis of acid-base balance and colloid osmotic structure. Besides its role as carrier to transport lipids, hormones, vitamins and minerals in the circulatory system, they often catalyze and regulate myriads of biochemical reactions at the cellular level (Tothova et al., 2016). Albumin plays an important role in maintaining homeostasis, in transport of substances (bilirubin, hormones, metals, vitamins, and drugs), binds fatty acids for improved fat metabolism, and acts as a freeradical scavenger (Tothova et al., 2016). Uric acid is being synthesized in the liver and excreted through the kidney, thus higher uric acid concentration might predispose the liver and kidney to hyperactivity and impairment (Adeyemo et al., 2000; Fafiolu et al., 2014). Liver enzymes (AST and ALT) are involved in protein metabolism and detoxification of potential harmful substances (Singh et al., 2011). This current finding agrees with the previous studies (Maroufyan et al., 2010; Fafiolu et al., 2014) that dietary nutrients influenced serum biochemical profile of broiler chickens. In addition, Awad et al. (2014) reported reduced serum total protein and uric acid when $16.20 \% \mathrm{CP}$ diet was fed to broiler chickens. Elevated level of globulin, uric acid, AST and ALT in broilers fed feeds stored in lit room might be attributed to increased immunoglobulin synthesis, inefficient protein utilization and physiological stress on liver and kidney. Higher creatinine level in broilers fed feed S stored in lit room could suggests depletion of muscle protein and/or impairment of protein utilization which could be attributed 


\section{Diet matrix and broiler chicken performance}

to the poor growth performance in such birds. Creatinine is a chemical waste molecule that is generated from muscle metabolism when creatinine phosphate is catabolized (Fafiolu et al., 2014).

Chickens' intestine functions majorly in the digestion of ingested feeds into constituent parts, absorption of dietary nutrients and act as a defense against enteric microbes (Burrin, 2002). Nutrient digestion and absorption is dependent on several factors including the size (and weight) of the GIT, feed form (mash, pellet or crumbles), particle size, secretion capacity of digestive juices and enzymes, as well as intestinal mucosa architecture such as villus height, villus surface area, and the rate of enterocytes turn-over (Zang et al., 2009; Laudadio et al., 2012). Higher VH and VCR in the duodenum and ileum of broilers fed feed $\mathrm{R}$ stored in dark room when compared with other diets indicated better nutrient absorption and decreased turnover rate of intestinal epithelium. Fafiolu et al. (2020) opined that higher VCR is an indication of lower rate of enterocyte-cell migration from the crypt to the villus. This implied that small proportion of the nutrient absorbed will be required for maintenance, and thus suggests better gut health in chickens. In the current study, significant variation in villi height or crypt depth across the small intestine due to feed brands effect agreed with the studies of Laudadio et al. (2012) who reported significant variation in morphology measurements of broiler chickens fed varying dietary nutrients. The structure of cereal grains and composition of exogenous enzymes used for broiler diets alongside with the digesta viscosity, passage time, optimal $\mathrm{pH}$ of enzymes, and functionality of GIT components could contribute to the effectiveness of digestion and absorption of nutrients in broiler chickens (Craig et al. 2019). Amerah et al. (2007) and Zang et al. (2009), when comparing the effects of feed particle size and feed form on gut morphology of broiler chickens, reported significant difference in villus height and villus height to crypt depth ratio.

Lower VH and VCR values obtained in broilers fed feeds stored in Lit room could be attributed to the reduced nutrient digestibility and growth performance as much of the dietary nutrients absorbed are being used for intestinal turnover and body maintenance. In addition, higher lymphocytes, serum globulin, uric acid, and liver enzymes recorded in such broilers could be adaptive responses to stress, and a confirmation that dietary nutrients meant for meat tissue deposit were diverted towards immunity and tissue maintenance. Earlier, Awad et al. (2009) had reported that stressors, presence of toxins and pathogenic microbes have deleterious effects on integrity of intestinal epithelium with resultant impairment of the metabolic processes. Furthermore, $\mathrm{Xu}$ et al. (2003) reported decline in growth performance, nutrient digestibility and reduced disease tolerance as the detrimental impact of alteration of intestinal morphology.

The significant role of chicken's gut microbiota in optimizing the gastrointestinal development and functions, efficient nutrient metabolism and utilization, as well as modulation of host immune system necessitates the need to support the proliferation of beneficial microbes through various dietary or managerial strategies (Pan and $\mathrm{Yu}, 2014$ ). Hind gut fermenters help to convert by-pass dietary components and endogenous gut secretions into high-energy metabolites which supply more energy in form of short chain fatty-acids for the host (Rinttilä and Apajalahti, 2013). They also increase the secretion and activity of intestinal digestive enzymes including amylase, protease, trypsin and lipase, enhance nitrogen 


\section{Fafiolu and Alabi}

metabolism towards de novo amino acids synthesis, and support supply of B-vitamins (Pan and Yu, 2014). In this current study, there was a feed type $\mathrm{x}$ storage interaction on microbial population in chickens' gut. Previous studies have shown that differences in diets composition, digesta retention time, metabolic stress, environmental factors including litter microbiome, as well as host's exposure to microbial inoculum at the early post-hatch days are the determinant factors to the microbial ecology in chickens (Rinttilä and Apajalahti, 2013; Alabi et al., 2018). Higher Salmonella counts in broilers fed feed $\mathrm{R}$ stored in lit room could be related to impaired performance as evident in reduced feed consumption, apparent nutrient digestibility, and higher serum uric acid and ALT levels in broilers fed such diets. Alabi et al. (2018) earlier noted that presence of toxigenic pathogens might cause feed refusal with resultant reduced growth efficiency, lower disease-tolerance, and induced pathologic damage to the liver. In addition, competitive exclusion might have been employed by commensals and Lactobacillus spp to minimize salmonella population in broilers fed other diets.

Increased Lactobacillus counts in broilers fed $\mathrm{S}$ stored in lit room could indicate higher lactic acid production from fermentation of undigested carbohydrates with resultant effects on $\mathrm{pH}$ reduction in the gut, inhibition of pathogenic bacteria and ultimately, improved gut health. Lactobacilli also stimulate synthesis of $\alpha$ - and $\beta$ galactosidase which enhance carbohydrate metabolism (Mountzouris et al., 2007). Noujaim et al. (2008) reported increase in lymphocytes and IgA production in the intestine of broiler chickens following Lactobacillus administration. Dietary supplementation of Lactobacillus acidophilus increased duodenal villus height and ileal villus height to crypt depth ratio in broilers (Pan and $\mathrm{Yu}, 2014$ ). The increase in clostridium counts recorded in broilers fed feed S may not necessarily suggest serious illness because majority of the Clostridia bacteria are beneficial to the animals as they are responsible for production of butyrate which supply energy for the enterocytes (Rinttilä and Apajalahti (2013).

The proportion of edible meat in a processed chicken depicts the carcass yield. It remains one of the economic production criteria that determine the gross margin of broiler production. Higher dressed yield percentage in broilers fed feed S stored in lit room further established the fact that availability of essential nutrients including amino acids (lysine, methionine and threonine), trace vitamins and minerals in adequate quantity are necessary for muscle tissue build-up and body maintenance (Alabi et al., 2017). Non-variation in carcass traits among broilers fed different feed brands agreed with the findings of Tavernari et al. (2014) and Muñoz et al. (2018) who reported that carcass yield was not influenced by the nutritional levels of the diets. In previous studies, Kamran et al. (2008) reported no significant different in all carcass yield and relative organs weights in broilers fed low-protein diets with constant energy-to-protein ratio. Besides the differences in treatment imposed, variation in diet composition, strain of broiler used, and rearing conditions could also be responsible for the discrepancies in carcass yield reported findings. Gizzard functions to grind the feed particle size for downstream digestion and enhancement of digestive secretions. Similar gizzard weight in this current study suggested no adverse effects on the digestibility of dietary components (Craig et al., 2019).

\section{Conclusion}

The results of this present study 
demonstrated that exposure of similar or different finished feeds to varying storage conditions would impact broiler's performance differently. Hence, the quality of broiler feeds should be maintained by poultry farmers and feed distributors through proper storage in order to optimize production performance and welfare of broiler chickens.

\section{Conflict of interest}

There is no personal, financial, or other form of conflict as regard the products or organizations mentioned in this paper. This research did not receive any specific grant from funding agencies in the public, commercial, or not-for-profit sectors. Proprietary finished feeds used (Premier Feeds, Animal Care Feeds, Hybrid Feeds, and Rainbow Feeds) are listed not in any particular order.

\section{Acknowledgement}

The authors acknowledge poultry farm staff and students who assist in data collection and sample preparation in the laboratory. The support received from the World Bank Centre of Excellence in Agricultural Development and Sustainable Agriculture, Federal University of Agriculture, Abeokuta, Nigeria is duly appreciated.

\section{References}

Adeleye, O. O., Otakoya, I. O., Fafiolu, A. O., Alabi, J. O., Egbeyale, L. T. and Idowu, O. M. O. 2018. Serum chemistry and gut morphology of two strains of broiler chickens to varying interval of post hatch feeding. Veterinary and Animal Science, 5: 20-25.

Adeyemo, G. O., Ologhobo, A. D. and Adebiyi, O. A. 2010. The effect of graded levels of dietary methionine on the haematology and serum biochemistry of broilers.
International Journal of Poultry Science, 9: 158-161.

Akit, H., Mohd Noor Zainudin, N. N., Abdul Wahid, N. A., Zakaria, S. N., Foo, H.L. and Loh, T. C. 2019. Dietary bromelain improves nutrient digestibility, digesta viscosity and intestinal villus height as well as reduces intestinal E. coli population of broiler chickens. Malaysian Journal of Animal Science, 22(1): 1-16.

Alabi, J. O., Fafiolu, A. O., Akande, F. A., Oluwatosin, O. O., Sogunle, O. M., Dada, I. D., Adeleye, O. O. and Olagoke, K. O. 2018. Assessment of microbial profile of selected proprietary broiler feeds sold in Abeokuta, South-West, Nigeria. Nigerian Journal of Animal Science, 20(1): 183-190.

Alabi, J. O., Fafiolu, A. O., Oso, A. O., Jegede, A. V., Dada, I. D., Teniola, A. A. and Oluwatosin, $O$. $O$. 2017. Physico-chemical and compositional changes in proprietary finished feeds stored under different conditions. Archivos de Zootecnia, 66(256): 535-541.

Amerah, A. M., Ravindran, V., Lentle, R. G. and Thomas, D. G. 2007. Influence of feed particle size and feed form on the performance, energy utilization, digestive tract development and digesta parameters of broiler starters. Poultry Science, 86: 2615-2623.

Association of Official Analytical Chemists (AOAC). 2000. Official Methods of Analysis. 17 $7^{\text {th }}$ Edition, Association of Official Analytical Chemists International Inc., Washington DC, USA.

Awad, E. A., Fadlullah, M., Zulkifli, I., Farman, A. S. and Chwen, L. T. 
2014. Amino acids fortification of low-protein diet for broilers under tropical climate: ideal essential amino acids profile. Italian Journal of Animal Science, 13:270-274.

Awad, W. A., Ghareeb, K., AbdelRaheem, S. and Bohm, J. 2009. Effects of dietary inclusion of probiotic and synbiotic on growth performance, organ weights, and intestinal histomorphology of broiler chickens. Poultry Science, 88: 49-55.

Burrin, D. G. 2002. Gastrointestinal protein and amino acid metabolism in growing animals. In: Biology of the intestine in growing animals. Zabielski R., Gregory PC., Welstrom B. (editors.). Elsevier Science B.V., Amsterdam, Netherlands.p. 695-725.

Craig, A. D., Bedford, M. R., Hastie, P., Khattaka, F. and Olukosi, O. A. 2019. The effect of carbohydrases or prebiotic oligosaccharides on growth performance, nutrient utilisation and development of small intestine and immune organs in broilers fed nutrient-adequate diets based on either wheat or barley. Journal of the Science of Food and Agriculture, 99: 32463254.

Fafiolu, A. O., Alabi, J. O., Godwin, C. G., Dada, I. D., Orimogunje, A. A., Osinowo, O. A., Bello, Z. O. and Ogunkanmbi, T. O. 2020. Phytogenics (Lemon and orange peels) influenced the performance, gut morphology and blood profile of broiler chickens. Nigerian Journal of Animal Production, 47(3): 234-244.

Fafiolu, A. O., Otakoya, I. O., Adeleye, O. O., Egbeyale, L. T., Alabi, J. O. and Idowu, O. M. O. 2014.
Comparing the blood profile of two strains of Broiler chickens with varying interval of post hatch feeding. Nigeria Journal of Poultry Science, 11: 196-203.

Horn, N. L., Radcliffe, J. S., Applegate, T. J. and Adeola O. 2010. Gut morphology and nutrient retention responses of broiler chicks and White Pekin ducklings to dietary threonine deficiency. Canadian Journal of Animal Science, 90: 513520 .

Kamran, Z., Sarwar, M., Nisa, M., Nadeem, M. A., Mahmood, S., Babar, M. E. and Ahmed, S. 2008. Effect of low-protein diets having constant energy-to-protein ratio on performance and carcass characteristics of broiler chickens from One to Thirty-five days of age. Poultry Science, 87: 468-474.

Laudadio, V., Passantino, L., Perillo, A., Lopresti, G., Passantino, A., Khan, R.U. and Tufarelli, V. 2012. Productive performance and histological features of intestinal mucosa of broiler chickens fed different dietary protein levels. Poultry Science, 91:265-270.

Lopez, C. A. A., Baião, N. C., Lara, L. J. C., Rodriguez, N. M. and Cançado, S. V. 2007. Effect of physical form of ration on digestibility and performance of broiler chickens. Arquivo Brasileiro Medicina Veterinária e Zootecnia, 59(4): 1006-1013.

Maroufyan, E., Kasim, A., Hashemi, S.R., Loh, T.C., Bejo, M.H. and Davoodi, H. 2010. The effect of Methionine and Threonine supplementation on immune responses of Broiler Chickens challenged with Infectious Bursal Disease. American Journal of 
Applied Sciences, 7(1): 44-50..

Mountzouris, K. C., Tsirtsikos, P., Kalamara, E., Nitsch, S., Schatzmayr, G. and Fegeros, K. 2007. Evaluation of the Efficacy of a Probiotic Containing Lactobacillus, Bifidobacterium, Enterococcus, and Pediococcus Strains in Promoting Broiler Performance and Modulating Cecal Microflora Composition and Metabolic Activities. Poultry Science, 86: 309-317.

Muñoz, J. A., Suckeveris, D., Demuner, L. F., Saccomani, A. P. O., Silva, A. L., Silva, R. T., Faria Filho, D. E. and Faria, D. E. 2018. Effects of nutritional levels on performance, carcass characteristics and nutrient digestibility of sexed broilers. Brazilian Journal of Poultry Science, 20(1): 053-062.

Noujaim, J. C., AndreattiFilho, R. L., Lima, E. T., Okamoto, A. S., Amorim, R. L. and Torres Neto, R. 2008. Detection of $T$ Lymphocytes in Intestine of Broiler Chicks Treated with Lactobacillus spp. and Challenged with Salmonella enterica Serovar Enteritidis. Poultry Science, 87: 927-933.

Orheruata, A. M., Nwokoro, S. O., Alufohai, G. O. and Omagbon, B. I. 2006. Growth Indices and economy of feed intake of broiler chickens fed changing commercial feed brands at starter and finisher phases. International Journal of Poultry Science, 5(12): 1123-1127.

Oso, A. O., Williams, G. A., Oluwatosin, O. O., Bamgbose, A. M., Adebayo, A. O., Olowofeso, O., Pirgozliev, V., Adegbenjo, A. A., Osho, S. O., Alabi, J. O., Li, F., Liu, H., Yao, K. and Xin, W. 2017.
Effect of dietary supplementation with arginine on haematological indices, serum chemistry, carcass yield, gut microflora, and lymphoid organs of growing turkeys. Livestock Science, 198: 58-64.

Oyediji, J. O., Umaigba, J. O., Okugbo, O. T. and Ekunwe, P. A. 2005. Response of broiler chickens to different dietary crude protein and feeding regimens. Brazilian Journal of Poultry Science, 7(3): 165-168.

Pan, D. and Yu, Z. 2014. Intestinal microbiome of poultry and its interaction with host and diet. Gut microbes, 5(1): 108-119.

Qureshi, M. A. 2003. Avian macrophage and immune response: An overview. Poultry Science, 82: 691698.

Reddy, D. V. 2001. Principles of Animal Nutrition and Feed Technology, 2nd edition. Oxford \& IBH Publishing Company Pvt, Ltd., New Delhi, India.

Rinttilä, T. and Apajalahti, J. 2003. Intestinal microbiota and metabolites - Implications for broiler chicken health and performance. Journal of Applied Poultry Research, 22: 647-658.

Sakomura, N. K. and Rostagno, H. S. 2016. Research methods in monogastric nutrition. 2nd edition. Jaboticabal, SP: Funep, Brazil.

SAS, 2007. Statistical Analysis System for Windows, 9.1.3 portable version. SAS Institute Inc., Cary, North Carolina, NC 27513, USA.

Singh, A., Bhat, T.K. and Sharma, O. P. 2011. Clinical Biochemistry of Hepatotoxicity. Journal of Clinical Toxicology, S4: 001. DOI: 10.4172/2161-0495.S4-001

Swaggerty, C. L., Pevzner, I. Y., Lowry, V. 
K., Farnell, M. B., Kogut, M. H. 2003. Functional comparison of heterophils isolated from commercial broiler chickens. Avian Pathology, 32(1): 95-102.

Talebi, A., Asri-Rezaei, S., Rozeh-Chai, R. and Sahraei, R. 2005. Comparative studies of haematological values of broiler strains (Ross, Cobb, Arbor-acres and Arian). International Journal of Poultry Science, 4(8): 573-579.

Tavernari, F. C., Bernal, L. E. P., Rostagno, H. S., Albino, L. F. T. and Vieira, R. A. 2014. Nutritional requirements of digestible methionine + cysteine for cobb broilers. Revista Ceres, 61(2): 193201.

Tothova, C., Nagy, O. and Kovac, G. 2016. Serum proteins and their diagnostic utility in veterinary medicine: a review. Veterinary Medicine, 61: 475-496.

Uchegbu, M. C., Igbokwe, N. O., Omede, A. A., Ekwuagana, I. C., Okoli, I. C. and Esonu, B. O. 2009. Performance evaluation of some commercial broilers finisher rations sold in Nigeria. . International Journal of Poultry Science, 8(12): 1162-1164.

Uppal, D. S., Ilyas, S. M. and Sikka, S. S. 2004. Quality and safety of Animal Feeds in India. United Nations Asian and Pacific Center for Agricultural Engineering and $\begin{array}{llllllllllllllll}M & a & c & h & i & n & e & & & y & \text {. }\end{array}$ http://www.unapcaem.org/activitie s\%20files/a16/animal $\% 20$ feed $\% 2$ 0\%26\%20quality.pdf Pp 30. [Date assessed: 16 October, 2015].

Virden, W. S. and Kidd, M. T. 2009. Physiological stress in broilers: Ramifications on nutrient digestibility and responses. Journal of Applied Poultry Research, 18: 338-347.

Waheed, A., Ahmad, T., Yousaf, A. and Zaefr, I. J. 2004. Effect of various levels of fat and antioxidant on the quality of broiler rations stored at high temperature for different periods. Pakistan Veterinary Journal, 24(4): 70-75.

Wakenell, P. S. 2010. Hematology of Chickens and Turkeys. In: Schalm's Veterinary Hematology. $6^{\text {th }}$ edition. Weiss D.J., Wardrop K.J. (ed.) A John Wiley and Sons Ltd Publication, Wiley-Blackwell Publisher, USA. p. 958-967.

Xu, Z. R., Hu, C. H., Xia, M. S., Zhan, X. A. and Wang, M. Q. 2003. Effects of dietary fructo-oligosaccharide on digestive enzyme activities, intestinal microflora and morphology of male broilers. Poultry Science, 82: 1030-1036.

Yegany, M., Butcher, G., Nilipour, A. H., Miles, R. D. and Karegar, R. 2002. Feed hygiene and poultry health. World Poultry-Elsevier, 18(7): 17-19.

Zang, J. J., Plao, X. S., Huang, D. S., Wang, J. J., Ma, X. and Ma, Y. X. 2009. Effects of feed particle and feed form on growth performance, nutrient metabolizability and intestinal morphology in Broiler chickens. Asian-Australian Journal of Animal Science, 22(1):107-112.

Received: $9^{\text {th }}$ July, 2020 Accepted: $5^{\text {th }}$ October, 2020 